


\section{apibrangy of the attursenm}

\section{OF \\ COMPARATIVE ZOÖLOGY,}

AT HARTARd COLLEGE, CAMBRidge, MLSS.

f)ounder by pribate subscríption, în 1861.

DR. L. DE KONINCK'S LIBRARY. No. 1792. 





\section{Fr. Ernesti Brḯckmanni,}

Medicin. Doctor. \& Practici Brunfivicenfis De

Fabulofiffimæ originis lapide,

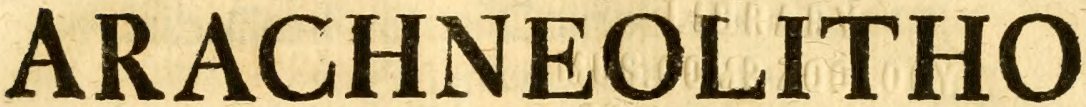

Dicto,

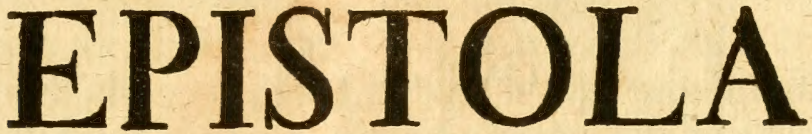

Ad

Virum Clariffimum atque Doctiffimum

AlBERTVM RITTERVM

Sub-ConRectorem cœnobialis Regii

Iycei, quod ad oras Hercynix floret,

Ilfeldenfis,

Mathematicum \& Phyficum

celeberrimum.

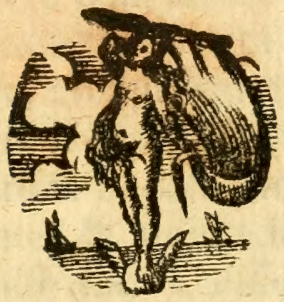

Wolfenbüttelx, Anno Chrifti MDCCXXII.

Sumptibus Joh. Chriftoph. Meisneri. 


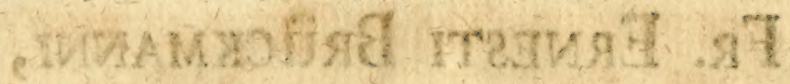

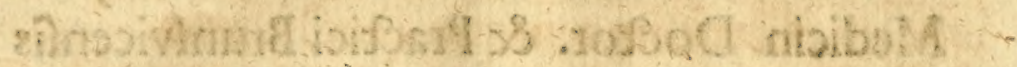
90

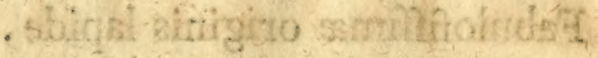

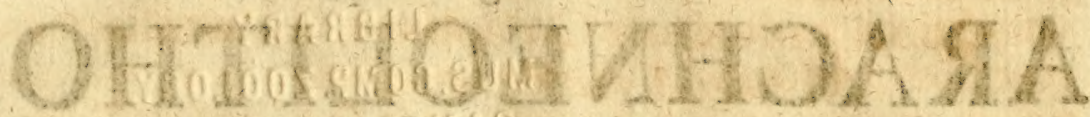
that की

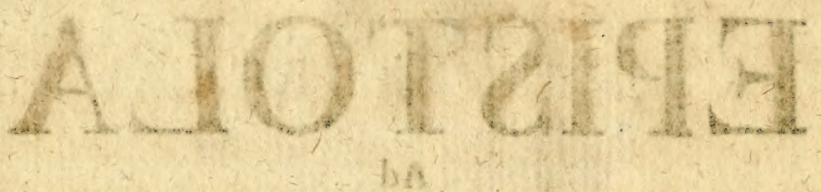

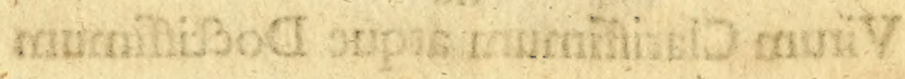
MVAATILA MVTRHEJA

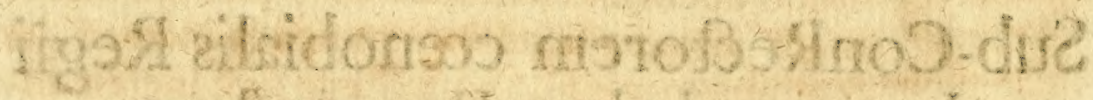

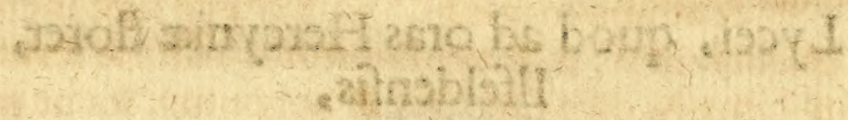

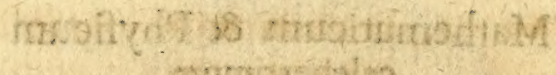

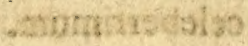

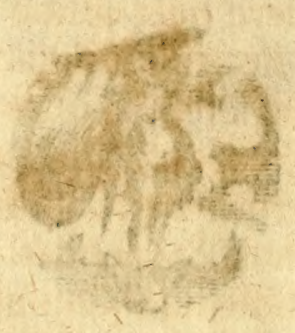

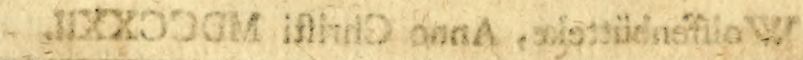

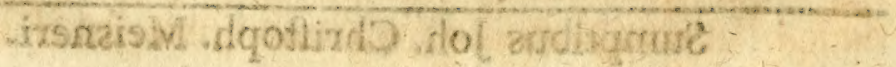




\section{VIR}

\section{Clariffimo atque Doctifimo,}

ALBERTO RTTTRRO,

Regii Lycei celeberrimo,

Mathematico \& Naturæ Ipeculatori curjofinimo, Salutem plurimam dicit

$$
\text { F. E. Br. }
$$

\section{I.}

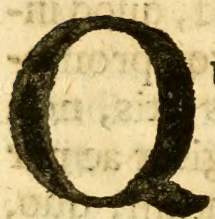

uemadmodum fuperiori feculo, Vir clariffime atque doctiffime, fcientia naturalis fua incrementa fumere haud contemnenda incœpit, ita $\&$ adhuc magis hoc noftro ævo hæc incremen: ta per celeberrimos \& magni nominis naturæ fcrutatores accumulantur in dies fingulos \& augentur, ita ut mox ad fummum faftigium, fummumque perfectionis culmen evecta videri poffit; Aft vero tämen rem cum paulo penitius intuemur, videre licet adhuc multa ei deefle, præ, fertim quoque cum nec unius vita faltim fufficiat ad delendas \& deftruendas, credula quas antiquitas in hanc divinam fcientiam ingredi \& illabi pafla fuit, fabulas. Circa nullam autem rerum naturalium partem copiofiores invenire licuit vanitates \& mendatia, quam in theoria lapidum, horumque hiftoria. Caufas hine nobis videmur habere non unas, de hoc argumento ad TE, Vir clariffime atque doctiffime, exarare epiftolam, \& perfcribere pauca. In confiderationem autem jam placet trahere la-

$$
\text { A } 2
$$

pidem 


\section{EPISTOLA DE ARACHNEOLITHO.}

pidem aranearum, cujus oniginem fabulofiftimam autores tradünt; Lapidem ipfum a nemine, fi mendacentrejts \& vanam excipias hiftoriam, pertractatum novinus. Operx igitur pretium nobis viflum eft, fin panca in chartam de hoc conjecta clariffimg T VO nomini nuncuparemus; Præxterquam enim, quod ante decennium fudiano: Itra humaniora per tres integros annos fideliter, qvare non æternas tantum TIBI habemus gratias, fed \& haud minimam felicitatis noftrx partem THB in acceptis referimus, imo fideliffime direxeris, \& deinde nobis abfentibus per literas, humanitatis pleniffimas, affectum TVVM in nos prolixum abundeteftatus fueris; Accedit \&illud, quod ingenti TE, ftudia \& qualefcunque conatus noftros promovendi flagrare ftudio ac defiderio, re ipfa plus fatis, nôn verbis tantum teftatus ompino fis; Aqquum igitur ac par effe judicavimus, publicum exftare monumentum, quo, quanti TE faciamus, quantoque amore \& fervore TVI optemus favoris perennitatem longiffimam, bonis omnibus; \& in primis clariffimi nominis TVI cultoribus, dut culentius paulo fignificaremus; Quod vero ut æqui bos nique confulas, gratoque ac benigno favore, quo nihil nobis carius vel gratius effe poterit, nos pariter atque prafentem ingenii noftri exigui foetum exiguum atque informem complectaris, nox minus certo fperamus, quam votis omnibus juftis contendimus.

$$
\text { S. II. }
$$

Hiftoriam noftri lapidis, quem in antecedentibus in confiderationem trahere promifimus, fabulofam effe diximus; Autorem vero, qui his, fabulis anfam dedit, invenire hon pottiunus, quam diligentiffime eciam veterum natu- 
naturalis hiftorix compilatorum libros \& fcripta evolverimus. Placet hinc primo variorum autorum monumenta de hoc lapide recenfere \&o deinde noftras addere de illo præmeditatas cogitationes, $\&$ ad quod lapidum figuratorum pertineat genus mentem aperire noftram.

\section{D.III.}

Primo in medium proferemus locum Chr. Fr. Paullini, qui in fuis horis amœnis philofophicis part. I. quæftion. LXII. p. 424. fequentem movet quæftionem: An poffibile fit aranearum lapidem adipifci \& quomodo? Ubi hunc in modum refpondet: Sin erften zwciffelt D. Simon Pauli, uno bålt ę füt lauter gabelwerck / tos. antoete von Spinnen: Stein erzeblen. Denn wie er fu 2 Bittenberg studitte / traff ex einft eine ungebeute groffe Spinte an/ fo

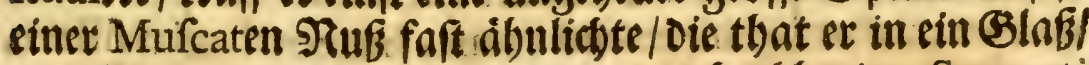
uno fúlte foldbes auf (Butad)ten oes berúbmten Sennerti fiemlich mit grob gepulberten groffen Baldrians-233urfel an/ in getviffet Soffnumg/ fie witde einen Stein fallen

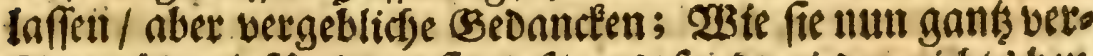
fomadstete / fontt er fie auf / uno fano wieder niddts bets ibr; Anvere legen unter ibt (Bewebe/ Dareit fie gemeinigs lich mitten fitst/geoadbte 23 urzel / aber mit gletdem Blite. Dod bat D. Joh. Francke bon 15 cingerpetrten Spinmen

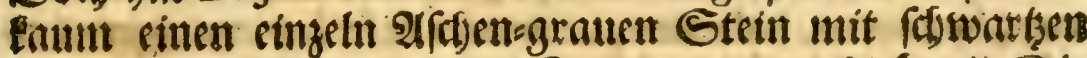
Bunctlein erlangen fonnen; Drim er gar tedt faget: Die wentgiten Spinnen bàtten Derglețen oấ alie Der gute D. Simon bon einer Spinne nuf alle obn unterfacio nidgte gewiffes bat fichliffen lonnen. Anoere wie geoactet Francke, ftecten Die Epinnen in ein mit gemetnen Ruddens Salt gefulltes Sefdoirt/ uno notbigen fie fo lange/ bitg fie A3

oen 


\section{EPISTOLA DE ARACHNIEOLITHO.}

Den Stein verzettelt. Dod gerdiths aud nict) affemabll

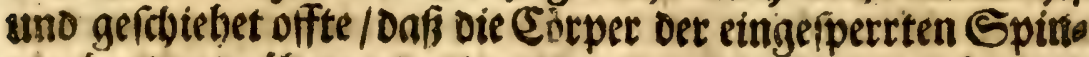
nen bart uno zăbe werden/ uno den Unwiffenden einen blouess Dunit borimablen/ weldae fie fur adbte Steine battents Denn ob gletch Dergleidgen Steine Dergleicben 23 irtetung

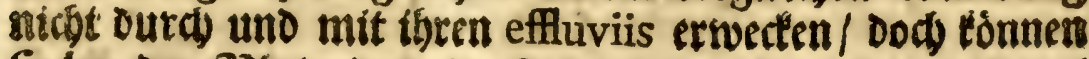
fie ben. Den 3 lutenden etme gutcht uno Sidouber erregen/

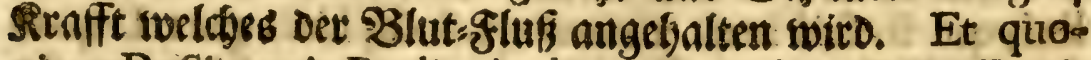
niam D. Simonis Pauli, Archiatri quondam Regii Danici celeberrimi, ab initio mentionem fecimus, ejus locum de hoc lapide addemus, qui in Botanic: Quadripart. Clafo fe II. Herbarum tempore verno florentium p.m. 179. 180. proftat. Vulgus perhibet araneas prægrandes lapillo foetas confpici, eumque ceu arcanum fummum \& fpecificum certum, inftar incantamenti omnes hæmorrhagias fiftere, modo is, manu ægri in pugnum colleetá, tamdiu foveae kur, ufque dum incalefcat, quem quoque non nulli ajunt, araneam evomere, excernere, parere, aut quocunque modo excutere, fi tela, quam texuit, \& in qua media has bitat, fubdideris Valerian $x$ radicem. Evenit autem, cum anno MDCXXX. ex Gallia redux, ftudiorum caufa Witrebergæ æftivarem, ibidem ex adverfo nunquam mihi fine veneratione nominando praceptore meo celebratiffmo, Sennerto, habitans, ut aranea difcolor crucigera impluvium mei hofpitis, Nicolai, Senatoris Wittebergenfis, obfediffer; Qux cum immenfx effet magnitudinis, æqua:bat certè nucleum nucis mofchatæ, non folum me, fed quoque Melchiorem Huffanff, tunc temporis Sennertidamefticum, operationum chymicarum peritiffimum, in fui adinirationem rapiebat; Convenimus nos inter nos, ca-

pimus 
pimus araneam, \& vitro vafto cucurbitino includimus, \& ex confilio communis noftri præceptoris Sennerti, ra dicibus Valerianæ perfunctorie pulverifatis, ex parte vicrum replemus, hac fpe certa freti, certo certius hane pragrandem araneam lapidem parturam, verum van laetati, ne atomum quidem ullam lapilliadipifcimur: Sed rerum naturalium ftudiofi \& veritatis cupidi emaciatam araneam difcindimus, at lapillo aut calculo nulla fui parte foetam; Fabulas ergo \& meras effe nugas, quæ de lapide aranearum vulgus perhibet, edoeti fumus. L. Ch. Hellwig inquit: 2Benn eine Spinne feben Jabr verfdloffer bliebe / folte fie zu einem Stein werden/ welder gat bod zu seftimiren und widoet Giff Diene/ uno ein Rancter s Steit genennet wutroe; Db tun abet Defer Steit / welcten etlicos in Ringe fesen lafen/auf oben gemelote stet entfebe / ear

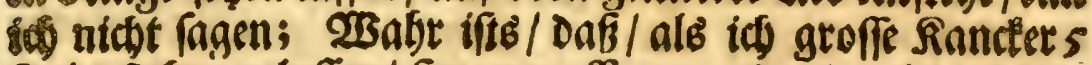
Jabe feben gelafien / fie zut maffa geworden/ welebe fo fert Dots man foldie Eaum fonetoen mogte/ Durch einen unver Rebenen Fall aber giengen oie Gláergen entzwet / uno wote ce vieletdt in Den lebsten zwev Jabren ote mafra feintgt uno fefter/ ja rectit fefe getworden; vide ejus netuangelegter curieufen und fut eDlen Medicin lurţ betfaften Thier: (Sats ten p. 286. Porro Gieffenfis Academiæ lumen, Dom. Mich. Bernh. Valentini de Arachneolitho ita differit: Go pfleges Der gemetne Mann aut vieles bon Den Spinnens. Stein ju fotwatsen/ tmo foldyen bor ein fonderlich Bifftstreibendes SYittel zu ritbmen/ uno weif mid zu erinnern/ Dafi ein bise fuger eadrtner befiwegen die groffen Rreuts: Stutuen tat Gdondteln nufgebolen I Damit er foldfen Strin exlongen

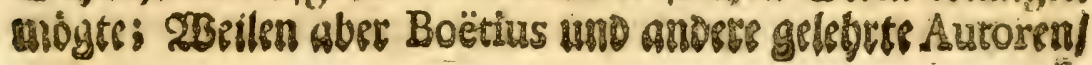




\section{EPISTOLA DE ARACHNEOLITHO.}

fo von alferbano Stetnen geforicben ocfea gat nicht ges Denten/ fo zweifele/ ob fict die Sache Damit alfo berbalte/ und ob focon neulid) bey einem guten greuno cinen Stein/ sworaut eine Spinne abgebildet war / gefeben / fo if poch now ungewif / ob ex bon einer Spinne getommen/ oder ale fo in oer (Frde gegeuget worden fey. vid. autoris citati gla turs und Materialien STrmmer part. I. pag. sI 6. Sub finem capitis de Tarantulis. Porro: (Eb bat oie Spinme autbero. nigen פtutsen in Der Medicin, uno follen ablonderlict) die

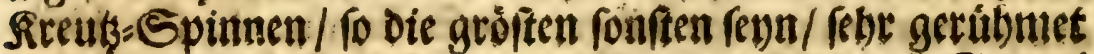
werden/ Dabero auch fommen roflen die Spinnen-Steine/ Denn wenn eine folde in ein Schädotlein getban / uno Jabe uno zag ontinnen verfdslofien gebalten wird/ fo verzebret fie

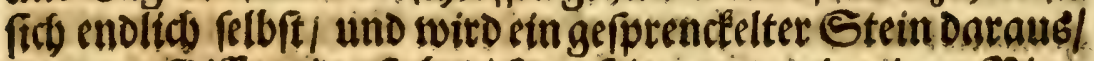
Der oem Gifft widerftebet / fo oaß̉ / suenn et in einen Rittg eingefabt an ginger gettragen wito/ er fo gleich die Garbe vernilloert / uno mit einer 230 lete úberziebet / wenn et ver

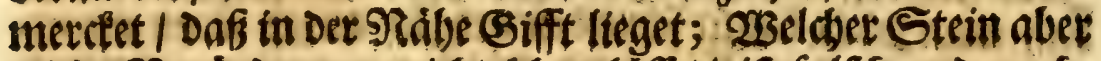

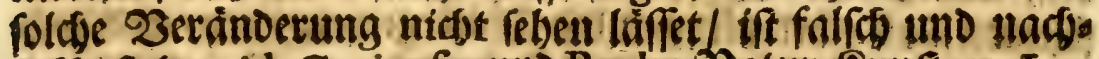

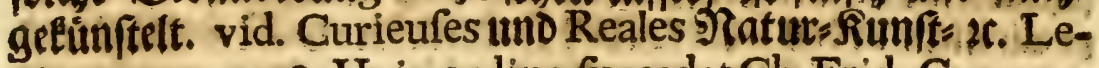
xicon pag. 1538. Huic ordine fuccedat Ch. Frid, Garmannus Lib. III. de miraculis mortuorum Tit. III. $\int: 85 . p$. 116. Arachnitem vix a quopiam fcriptore memoratum reperies, præter D. Joh, Francum, qui hujus exiftentiam probaturus aliquot experimenta in literas retulit, Ego, ut communis fert fabula, araneas maximas feptennium capfulis aliquoties inclufi, fed feptennio elapfo nihil præter pulverem quendam ex aranearum cadaveribus relictum confpexi \&c. Wolffius in fcrutinio amuletorum cap. II. fect. I. p. 192. inquit. dari lapidem ex araneis pe- 
endum contra hæmorrhagiam admodum a quibusdam commendatum, qui etiam certa ratione colligendus fit, quod tamen fabulofis nugis annumero \&cc. plura non adducemus loca, fuperfunt autem varia, referemus faltim hiftoriam de eo lapide, quam puer adhuc diverfis vicibus inaudivimus; Si nempe invenietur forfan prægrandis molis aranea, quæ vulgo apud nos in patria vocabulo Germanico, eine firtus Spime/ a fignatura forfan corporis externa, audit, \& conjiceretur in vitreum vas faccharo pulverifato refertum \& occluderetur, gravida lapide redderetur, quem quoque demum partu, vel vomitu ederet \& ab illo fe liberaret; Ufus haud contemnendi eum fore in fiftendis hæmorrhagiis, præterea etiam antiloimicum \& alexipharmacum.

$$
\text { f. IV. }
$$

Ex fupra allegatis variorum fcriptorum monumentis videre fatis fuperque licet, arachnitem effe lapidem generatum in infeeto quodam, quod apud nos aranea vel araneus audit, \& partu, purgatione per guttur fuperius vel inferius ejectum.

6. V.

Cum autem fecundum vulgi perfuafionem \& menrem arachnites ex aranea originem fuam habere $a b$ imperitis firmiffime credatnr, araneam, quale fit animalculum paulo analyfi fubjicere neceffe habemus exactiori; Refertur autem aranea vel araneus inter animalcula exfanguia minora feu infecta pedata, non alato, quod pre reliquis omnibus infectis ingeniofiffumum, ita, ut fapientiffimus quondam mortalium, Rex Salomo, araneam ad quatuor res minimas terræ, quæ fapientibus forent fapien- 
tiores numeret, lege ejus proverbia cap. XXX.v. 24. \& 28. Retia porro exitruit, quæ aranearum caflides \& telæ audiunt, fubtiliffima, quæ mulieres fane, quæ maxime ad nendi \& filum ducendi artificium, quod tamen araneis in acceptis referunt, digitorum argutiis valent, filumque elaboratiffimum conficere præclare fciunt, non cum eo fubtilitate operis funt conferendæ, hujus nimirum filum tenuitate pilum vincit. Præclare tenent araneæ texendi artem (textores eis fuam artem debent) \& natura funt ad geometriam eruditi (unde etiam variæ machinæ arachnes $\&$ araneæ nomine veniunt, quas vide in excellentiffimi Wolffii vocabulario mathematico) lineas enim, quas ducunt, omnes effe parallelas, \& integras telam conftituentes formam quandam horarii æquinoctialis expandere conftat, de qua aranearum geometria plura evolvere licet in Joh. Andr. Schmidii, Abbatis \& Polyhiftoris noftri xvi celeberrimi, cui ut Medicus ex Gilead priftinam renovet fanitatem \& cumulum annorum vitæ ejus addat in falutem orbis eruditi, omnium bonorum eft votum, variorum philofophicorum Decade Differtat. IX. de Geometria brutorum p.9.10. \&c. ubi \& icon hujus naturalisaranearum Geometriæ obfervanda. Ob fubtiliffima fila, quæ nent, fubtilitate digitorum eas pollere neceffe eft, imo tactu hominem præcellunt. Minutiffima porro entia vident, quæ vifum noftrum effugiunt, quia forfan in fubtiliore harum corpore fubtilius elaboratur \& generatur fluidum nervofum, quod fpiritus animales neoterici phyfiologorum appellare gaudent. Numero oculorum hominem \& reliqua animantium genera longe fuperant, quxdam enim fex, octo, decem \& adhuc pluribus inftrictæ funt 
funt oculis, qui hunc in modum in medio capite feu vertice $\because \because$ difpofiti noftris, microfcopio roboratis, oculis deprehenduntur, de quo oculorum numero \& ferie vid. Joh. Jacob. Wagneri Hiftor. natural. Helvetiæ curiofam p. m. 237. it. Borellum in centuria fua obfervationum microfcopicarum. Si ullum demum animalculum copiofam prolem edit, copiofaque ponit ovula, certe eft noftra aranea, qux plus femper quam fexcenta vel feptingenta in. lucem edere folet ovula, qua fua fponte demum abfque ulla incubatione ex in volucro fuo \& teftis prodeunt. Nunquam non medium telarum fuarum expanfarum occupant, hoftes, puto mufcarum familiam, jugulant \& cruore harum fe faginant. Antipathiam \& inimicitiam exercent perennem cum bufonibus, quos ictu fuo necant, de quo odio in bufones vide beatifími quondam mei præceptoris, quem virtus duobus abhinc menfibus fublimia vexit ad aftra, G. W. Wedelii, Medici confummatiffimi, viri omnium feculorum memoria digniffimi, æternæque apud omnem pofteritatem famæ, differtat. de Plantagine Jenæ 1712 . ventilatam;it. Plinium \& Henric. Ruyfchium in theatro univerfali ommium animalium Tom. III. p. 132. Araneæ omnes perhibentur effe hermaphroditæ, vid. Martini Schurigii fpermatologiam hiftorico-medicam p. 619. \& 682. it. Steph. Blancardum; it. Mifcell. Natur. Curioior. Dec. III. An. III. Obferv. LV.p. 65.ubi non araneæ tantum, fed totus infectorum cohors, fexus utriusque effe affirmantur. Integumenta fua feu pelles fre more omnium reliquorum ferme infectorum exuunt, vide Helmontium in operib. fub tittul. arcana Paracelfi p. m. 740. Magnitudo hujus animalculi variat, maxima araneæ fpecies in Brafi- 
lia, Nhamdù-Guaca dicta, pafferis magnitudine, tota tota pilis obfita, reperitur, cujus defcriptionem \& iconem vide apud Pifonem in Hift. Indiæ utriusque p. 284. qui citatus autor \& araneam Brafilienfem loco allegato humana facie argentei coloris æri incidendam curavit. Non omnes funt venenatæ, in numeræ enim proftant \& leguntur obfervationes, quod quidam fummo cum appetitu \& abfque ullo vitæ fubfequentidifcrimine eas devorarint; vide collectionem ejusmodi obfervationum in D. Heucheri araneo homini perniciofo \& falutari $\S$. 30. Venenum Rofa eis fug. gerere \& fuppeditare fcribitur. De ufu aranearum, earumque caffidum \& telarum falutari, medico \& noxio, cum epiftola horum recenfione limites haud difficile transgredi poffet, nihil hic inferere pro tempore placet, cum plura de his videri poterunt apud Antonium Thylefium in tractat. de araneola \& cicindela; Martinum Lifterum traetat. de araneis; fupra citatum Heucherum diff. de araneo homini perniciofo \& falutari; Kirchmaierum de aranea; Aldrovandum lib de infectis; Th. Moufetum minimorum animalium theatro; Henric. Ruyfch theatro univerfal. 0 mnium animalium \& alios; Anatomicam videre licet delineationem in Mifcell. Nat. Curiof Dec. II. An. II. p. 5 I. \& Dec. II. An. VII. p. 100. \& Mich. Bernh. Valentini amphitheat.Zootomic. fect. CXXIV. p. 190. de telis autem aranearum vid. Anonymi curieufe SRacbticbt von einet meuen 2rt Sefoe/ weldse von den Spimerweben zubereitet wited / und dabon der fónig in Frantetetd) Ludovicus XIV. sine $2 B$ efte gettrigen 36 .

f. VI.

Delineationem animalculi, ex quo Arachnites, per- 
brevem dedimus; Arachnitem autem ipfum nunc paulo penitius infpiciemus; nomen habet, uti vocabulum, quod Helladen fapit, ftratim indicat ex $\alpha^{\prime} \rho \alpha^{\prime} \chi$ ун , quod \& araneam \& puellam quandam Lydiam, argutiis digitorum pollentem, lanificii peritiffimam, qua ægre tulit opus fuum afpectu delicariffimum a Dea Minerva, qua cum certamen fufceperat, confractum \& ex invidia dilaceratum, laqueo gulam fibi fregit, vitamque cum morte commutavit, poft ab eadem Pallade, fua inimica, in araneam mutata, uti copiofioribus verbis hæc fabula \& metamorphofis apud Ovidium Lib. VI. fab. 4. exitat, fignificat. Latinis lapis aranearum idem eft, quod Græcis Arachnites. Placuit nobis vocabulũ quoddam, quod totum totũ Græcum, nempe áp ax no-

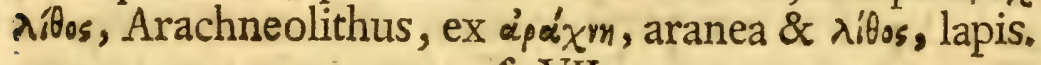

\section{f. VII.}

Intelligunt autem fcriptores per Arachneolithum lapidem $a b$ araneis defumtum, qui non confundendus cum araneis petrifactis \& lapideis, qualem Carol. Nic.Langius Hiftor. lapid. figurator. Helvetia Tab. X. p. 47. depingi

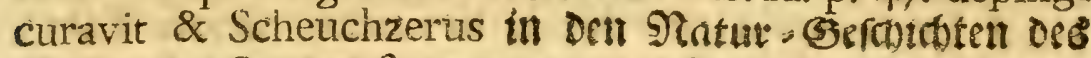
Edisther=\&andes fig. 179. p. 336. ad infecta diluviana re. fert. Nec quoque confundendus cum lapidibus, qui figuram \& iconem araneæ exhibent, qui quoque ad diluviana pertinent, de quibus vide jam femelallegatum Mich.Bernh. Valentini in Mufei Mufeorum part.I. p. 516.\& Scheuchzert in altera phyficæ parte p. 239. Eே f Strime/ast weldon gleidfan nad oem Eeven nbgemablet

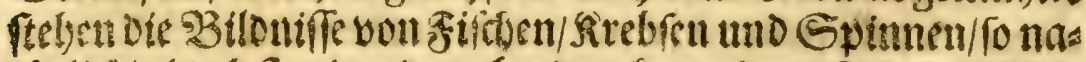

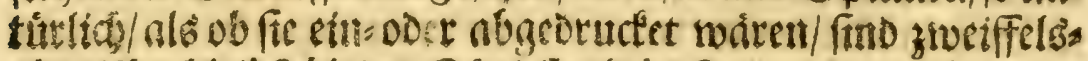

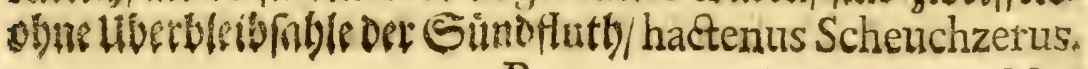

$$
\text { B } 3
$$

Nec 
Nec porro confundendus cum fuccino Pruffico, quo fæpius obductas \& naturali balfamatione æeternifatas vidimus araneas, de quibus Joh. Conr. Dannhauer hæc profert verba: Quod electro tali inclufæaëre deftitutæ durabiliores fint, quam Rex in fuo Maufoleo; vide quoque Rob. Boyle Hift. fluidor. \& folidor.p. I44. omnes hac paragrapho recenfiti lapides optimo jure nomen lapidum aranearum, oer Spitt: nen= ooet Ranclet=Steine / merentur, confufionis, tamen evitandæ gratia hæc afferre neceffum duximus.

\section{VIII,}

Icon adjacens Arachneolithum TIBI, Vir clariffime atque doctiffime, exhibet; Credimus autem TE ftatim, fimulac infpexeris modo paulo penitius iconem, dubitaturuim, lapidem nunquam in aranei vel araneæ corpore fuiffe, multo minus ab ejusmodi animalculo excretum five per os, five per alvum, five per alia corpufculi emunctoria; Magnitudo enim \& pondus ejus omnes ferme araneas, excepta Brafilienfi Nhamdu-Guaca dicta, longe fuperat; Arachneolithus autem, quem videre poteris apud Joh. Henr. Lochnerum inter Mulei Besleriani rariora Tabul. XXXV.p. 100. omnes araneas, tarantulas, phalangia \& Nhamdi-Guaca, gaudeant quoquo velint nomine ponde re \& magnitudine longe lateque excedit \& fuperat.

\section{S. IX.}

Noftro autem judicio Arachneolithus nihil aliud eft, quam corallium quoddam antidiluvianum petrifactum, quod Aftroiten Indicum nominare folent, \& pro certo credimus \& affirmamus, cum major aranearum pars elegantiffimis in abdomine maculis exornata deprehendatur, opinionem \& fabulam inde natas, quibus creduli perhibue- 
runt, originem ejus effe animalem; vel quoque a figura \& exteriori lapidis forma, quæ quandog figuran \& formam corporis aranearum detruncatis pedibus aliquo modo $x$ mulantur; quam tamen formam rariffime vel nunquam naturalem, femper potius adfcititiam \& artefactam putamus. I. X.

Aftroiten autem optimo jure appellare poffumus, plures enim elegantia confpicuæ ftellulæ in eo apparent, quæ non in fuperficie tantum lapidis apparent, fed per totum corpus lapideum tranfeunt. Dicitur Aftroites ab etymo Græco, quod eft äรpoy, fidus vel ftella, unde \& melius Aftritem, quam Aftroitem dicendum effe cenfemus. Non confudendus cum Aftroite Gemma, quæ pelluciditate nitet, cum nofter e contrario opacus fit lapis; Nec cum Afteria lapide, qui ftellam radiis angulofis repræfentat. Porro Indicum effe diximus, a patria nempe \& loco natali, omnium enim elegantiffimum India Orientalis nobis offert \& porrigit; Reperitur quoque \& proftat apud Helvetios, colligitur etiam apud Norimbergenfes \& Tyrolenfes \& apud diverfas alias gentes, qui tamen omnes elegantia ftellularum Indico noftro multum cedunt \& poltponuntur. Diverfæe ejus colliguntur fpecies, quæ vero, cum non fint hujus loci, tempus in iis recenfendis confumere nolumus, poffunt autem evolvi \& videri apud Bajerum, Langium, Luidium, Boetium a Boot, Sheuchzerum, Valentini, Büttnerum, Lachmundum, Mylium, Volckmannum, Hermannum, Lochnerum \&c. \& multos alios. Incubiculo fu. fpenfum araneos infectaque alia pellere \& fugare credunt creduli, faniores autem rem fictam effe putant, dum experimentum minime procedit.

S.XI. 
Nunc nos convertamus ad lapidem noftrum, quem depictum in adjacente tabula vides, ille natales fuos Orienti debet, opacus eft, egregie fplendens \& politus, quam tamen polituram arte nactam pro certo confidimus, durus, coloris albefcentis, egregiis optimeque figuratis ftellulis nuffefcentibus notatus, latere fuperiore convexus, inferiore vero feu averfo planus \& oviformem exhibet oculis ro tunditatem. f. XII.

Sed præter opinionem literæ hæ noftræ limites epiftolæ transgreff 2 videntur, quare opus, ut nunc finem eis imponamus, multa quidem adhuc reftant dicenda de hoc lapide, fed cum fatis fuperque apud nos conftet, \& animum TVVM in nos benevolum \& amicum probe femper cognoverimus, ea, quæ defunt, TE facile, Vir clariffime atque doctiffime, ex optime inftructa \& felecta bibliotheca TVA, cujus, quando recordamur, nunquam aliter fit, quam fumma cum mentis exhilaratione \& lætitia, fupplere $\&$ addere poffe, Quod reliqum eft, Vir clariffime atque doctiffime, ut ex voto bene reeteque yaleas, fifque maneasque conftanter, ut olim, ita \& porro literarum mearum fautor, vovemus \& devote precamur. Vale falvus \& in columis, favoreque TVO perenni me bea, qui ero, donec ero, perpetuus TVVS difcipulus. Dabam Brunfvigæ e Muféo meo d. 8. Jan, 1722.

\section{Explicatio figurarum.}

Fig. I. ) Siftit Arachneolithi vel rectius Aftritis latus inferius, quod planum atque politum eft.

Fig. II. ) Exhibet Aftritis latus fuperius, quod politum eft \& con. vexitate gaudet.

$$
\text { F I I I } \mathrm{S} \text {. }
$$




Do Not CIRalaAtE 
